\title{
A New Approximation Method for Finding Zeros of Maximal Monotone Operators
}

\author{
Vu Thi Ngoc \\ Hanoi University of Science and Technology, Hanoi, Vietnam \\ Email: Ngoc.vt211351M@sis.hust.edu.vn
}

\begin{abstract}
One of the major problems in the theory of maximal monotone operators is to find a point in the solution set $\operatorname{Zer}(\mathcal{A})$, set of zeros of maximal monotone mapping $\mathcal{A}$. The problem of finding a zero of a maximal monotone in real Hilbert space has been investigated by many researchers. Rockafellar considered the proximal point algorithm and proved the weak convergence of this algorithm with the maximal monotone operator. Güler gave an example showing that Rockafellar's proximal point algorithm does not converge strongly in an infinite-dimensional Hilbert space. In this paper, we consider an explicit method that is strong convergence in an infinite-dimensional Hilbert space and a simple variant of the hybrid steepest-descent method, introduced by Yamada. The strong convergence of this method is proved under some mild conditions. Finally, we give an application for the optimization problem and present some numerical experiments to illustrate the effectiveness of the proposed algorithm.
\end{abstract}

Keywords: Zero points, variational inequalities, maximal monotone operators, strongly monotone operators, lipschitz continuous operators.

\section{Introduction}

Let $\mathcal{H}$ be a real Hilbert space, $\mathcal{A}$ be a setvalued operator of $\mathcal{H}$ into $2^{\mathcal{H}}$ with domain $\mathcal{D}(\mathcal{A})=\{x \in \mathcal{H} \mid \mathcal{A}(x) \neq \varnothing\} \quad, \quad$ range $\quad \mathcal{R}(\mathcal{A})=$ $\bigcup_{x \in \mathcal{D}(\mathcal{A})} \mathcal{A} x$, and the inverse of $\mathcal{A}$ is $\mathcal{A}^{-1}(y)=\{x \in \mathcal{H} \mid y \in \mathcal{A}(x)\} . \quad \mathcal{A}$ is said to be monotone operator if

$$
\langle u-v, x-y\rangle \geq 0
$$

for all $x, y \in \mathcal{D}(\mathcal{A}), u \in \mathcal{A}(x), v \in \mathcal{A}(y)$

$\mathcal{A}$ monotone operator $\mathcal{A}$ is maximal monotone operator if the graph

$$
\mathcal{G}(\mathcal{A})=\{(x, y) \in \mathcal{H} \times \mathcal{H} \mid x \in \mathcal{D}(\mathcal{A}), y \in \mathcal{A}(x)\}
$$

of $\mathcal{A}$ is not properly contained in the graph of any other monotone operator in $\mathcal{H}$. For a monotone operator $\mathcal{A}$, we define its resolvent $\mathcal{J}_{r}^{\mathcal{A}}:=\left(I^{\mathcal{H}}+r \mathcal{A}\right)^{-1}$ by

$$
\mathcal{J}_{r}^{\mathcal{A}}: \mathcal{R}\left(I^{\mathcal{H}}+r \mathcal{A}\right) \subset \mathcal{H} \rightarrow \mathcal{D}(\mathcal{A}), r>0,
$$

where $I^{\mathcal{H}}$ being the identity map on the space $\mathcal{H}$.

One of the major problems in the theory of maximal monotone operators is to find a point in the solution set $\operatorname{Zer}(\mathcal{A})=\{x \in \mathcal{D}(\mathcal{A}) \mid 0 \in \mathcal{A} x\}$ where $\mathcal{A}$ is a set-valued operator of $\mathcal{H}$ into $2^{\mathcal{H}}$ with domain $\mathcal{D}(\mathcal{A})=\{x \in \mathcal{H} \mid \mathcal{A}(x) \neq \varnothing\}\}, \mathcal{H}$ be a real Hilbert space. This problem includes, as special cases, convex programming, variational inequalities, split feasibility problem, and minimization problem. The problem of finding a zero of maximal monotone in Hilbert spaces is investigated by many researchers.

For finding a zero of a maximal monotone operator $\mathcal{A}$, Rockafellar [1] considered the proximal point algorithm and proved the weak convergence of this algorithm. In order to have strong convergence, we have to modify this algorithm. Several authors proposed modifications of Rockafellar's proximal point algorithm to have strong convergence.

In this paper, our aim is to find a common zero of a finite family of $N$ maximal monotone operators $\mathcal{A}_{i}$, $i=1,2, \ldots, N$ in a real Hilbert space $\mathcal{H}$, i.e., an element of the set

$$
\mathcal{C}:=\bigcap_{i=1}^{N} \operatorname{Zer}\left(\mathcal{A}_{i}\right)
$$

We know that $\operatorname{Zer}(\mathcal{A})$ is a closed and convex subset of $\mathcal{D}(\mathcal{A})$ (see [2]). Therefore $\operatorname{Zer}\left(\mathcal{A}_{i}\right)$ is closed and convex for each $i=1,2, \ldots, N$. Hence $\mathcal{C}$ is closed and convex too. 
Let $\mathcal{F}: \mathcal{H} \rightarrow \mathcal{H}$ be a operator. $\mathcal{F}$ is said to be $\eta$-strongly monotone and $L$-Lipschitz continuous operator on $\mathcal{H}$ when the following conditions are satisfied

$$
\langle\mathcal{F} x-\mathcal{F} y, x-y\rangle \geq \eta\|x-y\|^{2}
$$

and

$$
\|\mathcal{F} x-\mathcal{F} y\| \leq L\|x-y\|
$$

for all $x, y \in \mathcal{H}$, where $\eta$ and $L$ are some positive constants. In addition, if $L \in[0,1)$, then $\mathcal{F}$ is called to be contractive and if $L=1$, then $\mathcal{F}$ is called to be nonexpansive.

The theory of Variational Inequality Problem (VIP) is well known, developed and appears to be one of the most important aspect in optimization and nonlinear analysis, since most mathematical problems can be modelled as a variational inequality problem. Let $\mathcal{C}$ be a nonempty, closed and convex subset of a real Hilbert space $\mathcal{H}$ and $\mathcal{F}: \mathcal{H} \rightarrow \mathcal{H}$ be a nonlinear operator. The (VIP) defined for $\mathcal{C}$ and $\mathcal{F}$ is to find $x_{*} \in \mathcal{C}$ such that $\left\langle\mathcal{F} x_{*}, x-x_{*}\right\rangle \geq 0 \forall x \in \mathcal{C}$.

In 2001, Yamada [3] introduced the hybrid steepest-descent method

$$
x_{k+1}=\left(I-\alpha_{k} \mu \mathcal{F}\right) \mathcal{T}_{[k+1]}\left(x_{k}\right),
$$

where $\mathcal{T}_{[k]}=\mathcal{T}_{k \bmod N}$ the $\bmod$ function taking values in the set $\{1,2, \ldots, N\}, \mathcal{T}_{i}: \mathcal{H} \rightarrow \mathcal{H}, i=1,2, \ldots, N$, is a nonexpansive mapping on $\mathcal{H}$ with $\mathcal{C}:=\bigcap_{i=1}^{N} \operatorname{Fix}\left(\mathcal{T}_{i}\right) \neq \varnothing$ and

$$
\begin{aligned}
\operatorname{Fix}\left(\mathcal{T}_{N} \ldots \mathcal{T}_{2} \mathcal{T}_{1}\right)= & \operatorname{Fix}\left(\mathcal{T}_{1} \mathcal{T}_{N} \ldots \mathcal{T}_{2}\right)=\ldots \\
& =\operatorname{Fix}\left(\mathcal{T}_{N-1} \ldots \mathcal{T}_{1} \mathcal{T}_{N}\right)
\end{aligned}
$$

and the parameter $\left\{\alpha_{k}\right\}$ satisfies the following conditions.

(C1) $\left\{\alpha_{k}\right\} \subset(0,1), \lim _{k \rightarrow \infty} \alpha_{k}=0$, and $\sum_{k=1}^{\infty} \alpha_{k}=\infty$.

(C2) $\sum_{k=1}^{\infty}\left|\alpha_{k}-\alpha_{k+N}\right|<\infty$.

In the present article, we will study a class of the variational inequality problem (VIP) with $\mathcal{C}$ is the set of common zeros of a finite family of maximal monotone operators in a real Hilbert space (CZP) by using the steepest-descent method and a modification of the algorithm of Ceng et al. [4] in a real Hilbert space. We introduce a new iterative method and prove the strong convergence of the presented method under some mild conditions.

The rest of this paper is divided into some sections. In Section 2, we recall some lemmas that will be used in the proof of our main theorems. In Section 3 , we present a method to construct approximate solutions. The last section we consider an example of numerical expressions.

\section{Preliminaries}

We have the following lemmas.

Lemma 1. Let $\mathcal{H}$ be a real Hilbert space and let $\mathcal{F}: \mathcal{H} \rightarrow \mathcal{H}$ be an $\eta$-strongly monotone and $L$ Lipschitz continuous operator on $\mathcal{H}$ with some positive constants $\eta$ and L. Then, for an arbitrarily fixed $\mu \in\left(0,2 \eta / L^{2}\right)$ and any $t \in(0,1), \quad I^{\mathcal{H}}-t \mu \mathcal{F}$ is a contraction with contractive constant $1-t \tau$, where $\tau=1-\sqrt{1-\mu\left(2 \eta-\mu L^{2}\right)}$.

(see [3])

Lemma 2. Let $\mathcal{C}$ be a nonempty closed convex subset of a real Hilbert space $\mathcal{H}$ and $\mathcal{T}: \mathcal{C} \rightarrow \mathcal{C}$ be a nonexpansive operator with $\operatorname{Fix}(\mathcal{T}) \neq \varnothing$. If $\left\{x_{k}\right\}$ is a sequence in $\mathcal{C}$ converging weakly to $x^{*}$ and if the sequence $\left\{\left(I^{\mathcal{H}}-\mathcal{T}\right) x^{k}\right\}$ converges strongly to $y$, then $\left(I^{\mathcal{H}}-\mathcal{T}\right) x^{*}=y ;$ in particular, if $y=0$, then $x^{*} \in \operatorname{Fix}(\mathcal{T})$, where $I^{\mathcal{H}}$ is the identity operator on $\mathcal{H}$.

(see [5], Lemma 2)

Lemma 3. $\mathcal{H}$ is uniformly convex if and only if, for each $\beta>0$, there exists a continuous strictly increasing and convex function $\varphi: \mathbb{R}^{+} \rightarrow \mathbb{R}^{+}$with $\varphi(0)=0$ such that

$$
\begin{aligned}
\left\|\alpha x_{1}+(1-\alpha) x_{2}\right\|^{2} \leq \alpha\left\|x_{1}\right\|^{2} & +(1-\alpha)\left\|x_{2}\right\|^{2} \\
& -\alpha(1-\alpha) \varphi\left(\left\|x_{1}-x_{2}\right\|\right),
\end{aligned}
$$

for all $x_{1}, x_{2} \in \mathcal{H}$ with $\max \left\{\left\|x_{1}\right\|,\left\|x_{2}\right\|\right\} \leq \beta$ and $\alpha \in[0,1]$.

(see [6])

Lemma 4. Let $\mathcal{H}$ be a real Hilbert space. If $\mathcal{B}: \mathcal{H} \rightarrow \mathcal{H}$ is a maximal monotone operator, then $\mathcal{I}_{\beta}^{\mathcal{B}}$ is non-expansive, single-valued mapping and $\operatorname{Fix}\left(\mathcal{I}_{\beta}^{\mathcal{B}}\right)=\mathcal{B}^{-1}(0)$ for each $\beta>0$.

(see [7], Section 7)

Lemma 5. Let $\mathcal{B}$ be a set-valued, monotone operator of $\mathcal{H}$ into $2^{\mathcal{H}}$. Then, for $r \geq s>0$, we have

$$
\left\|x-\mathcal{J}_{s}^{\mathcal{B}} x\right\| \leq 2\left\|x-\mathcal{J}_{r}^{\mathcal{B}} x\right\|
$$

for all $x \in \mathcal{R}\left(I^{\mathcal{H}}+r \mathcal{B}\right) \cap \mathcal{R}\left(I^{\mathcal{H}}+s \mathcal{B}\right)$.

(see [7], p. 42, [8], Lemma 2.2) 
Lemma 6. Let $\left\{w^{k}\right\}$ be a sequence of real numbers. Assume $\left\{w^{k}\right\}$ does not decrease at infinity, that is, there exists at least a subsequence $\left\{w^{k_{i}}\right\}$ of $\left\{w^{k}\right\}$ such that $w^{k_{i}}<w^{k_{i}+1}$ for all $i \geq 0$. For any $k \geq k_{0}$ define an integer sequence $\{v(k)\}$ as

$$
v(k)=\max \left\{0 \leq \mathrm{i} \leq k: w^{k_{j}}<w^{k_{j}+1}\right\} .
$$

Then $v(k) \rightarrow \infty$ as $k \rightarrow \infty$ and for all $k \geq k_{0}$

$$
\max \left\{w^{v(k)}, w^{k}\right\} \leq w^{v(k)+1}
$$

(see [9])

Lemma 7. Let $\left\{s_{k}\right\}$ be a sequence of nonnegative numbers satisfying the condition

$$
s_{k+1} \leq\left(1-b_{k}\right) s_{k}+b_{k} c_{k}, k \geq 0,
$$

where $\left\{b_{k}\right\},\left\{c_{k}\right\}$ are sequences of real numbers such that

(i) $\left\{b_{k}\right\} \subset(0,1)$ and $\sum_{k=1}^{\infty} b_{k}=\infty$,

(ii) $\quad \limsup _{k \rightarrow \infty} c_{k} \leq 0$.

Then, $\lim _{k \rightarrow \infty} s_{k}=0$.

(see [10], Lemma 2)

\section{Main Results}

In this section, we use the steepest-descent method and a modification of the algorithm of Ceng et al. in [4] to establish the strong convergence of the proposed algorithms for finding the solution of the problem (CZP), which is the unique solution of the (VIP). We assume that the solution set $\mathcal{C}$ of the problem (CZP) is nonempty. Hence, it is a closed convex subset of $\mathcal{H}$. Our algorithm can be expressed as follows.

\section{Algorithm 1.}

Step 0. Choose $\mu \in\left(0,2 \frac{\eta}{L^{2}}\right)$, the sequence $\left\{\alpha_{k}\right\}$ satisfies the condition $(\mathrm{C} 1)$, the sequences $\left\{\beta_{k}^{i}\right\}$ and $\left\{\gamma_{k}^{i}\right\}$ satisfy the following conditions

(C3) $\min _{i}\left\{\inf _{k}\left(\beta_{k}^{i}\right)\right\} \geq \beta>0$, and

(C4) $\left\{\gamma_{k}^{i}\right\} \subset[a, b] \subset(0,1) \forall i=1,2, \ldots, N$.

Step 1. Let $x_{0} \in \mathcal{H}$. Set $k:=0$.

Step 2. For all $i=1,2, \ldots, N$, compute

$$
z_{k}^{i}=\left(1-\gamma_{k}^{i}\right) z_{k}^{i-1}+\gamma_{k}^{i} \mathcal{J}_{\beta_{k}^{i}}^{\mathcal{A}_{i}} z_{k}^{i-1}, z_{k}^{0}=x_{k} .
$$

Step 3. Compute $x_{k+1}=\left(I^{\mathcal{H}}-\alpha_{k} \mu \mathcal{F}\right) z_{k}^{N}$.

Step 4. Set $k:=k+1$ and go to Step 2 .
Theorem 1. Let $\mathcal{H}$ be a real Hilbert space. Assume that $\mathcal{F}: \mathcal{H} \rightarrow \mathcal{H}$ is an $\eta$-strongly monotone and $L$ Lipschitz operator. Let $\mathcal{A}_{i}: \mathcal{H} \rightarrow 2^{\mathcal{H}}, i=1,2, \ldots, N$, be maximal monotone operators such that

$$
\mathcal{C}=\bigcap_{i=1}^{N} \operatorname{Zer}\left(\mathcal{A}_{i}\right) \neq \varnothing
$$

Then, the sequence $\left\{x_{k}\right\}$ generated by Algorithm 1 converges strongly to the unique solution of the (VIP). Proof Theorem. The proof consists in three steps.

Step 1. We will show that there exists a positive constant $M_{1}$ such that $\left\|x_{k}\right\|,\left\|z_{k}^{i}\right\|$ and $\left\|\mathcal{F}_{k}^{i}\right\| \leq M_{1}$ for all $k \geq 0$ and $i=1,2, \ldots, N$.

It follows from the condition (C3) and Lemma 4 that $\mathcal{J}_{\beta_{k}^{N}}^{\mathcal{A}_{N}} z=z$ for any point $z \in \mathcal{C}$. From the nonexpansive property of $\mathcal{J}_{\beta_{k}^{N}}^{\mathcal{A}_{N}}$, the property of the convex function $\|\cdot\|$, and Step 2 in Algorithm 3.1, we have that

$$
\begin{aligned}
& \left\|z_{k}^{N}-z\right\| \\
= & \left\|\left(1-\gamma_{k}^{N}\right)\left(z_{k}^{N-1}-z\right)+\gamma_{k}^{N}\left(\mathcal{J}_{\beta_{k}^{N}}^{\mathcal{A}_{N}} z_{k}^{N-1}-z\right)\right\| \\
\leq & \left(1-\gamma_{k}^{N}\right)\left\|z_{k}^{N-1}-z\right\|+\gamma_{k}^{N}\left\|\mathcal{J}_{\beta_{k}^{N}}^{\mathcal{A}_{N}} z_{k}^{N-1}-z\right\| \\
\leq & \left(1-\gamma_{k}^{N}\right)\left\|z_{k}^{N-1}-z\right\|+\gamma_{k}^{N}\left\|z_{k}^{N-1}-z\right\| \\
= & \left\|z_{k}^{N-1}-z\right\| \\
\vdots & \leq z_{k}^{0}-z\|=\| x_{k}-z \| .
\end{aligned}
$$

It follows from Step 3 in Algorithm 1, (3), and Lemma 1 that

$$
\begin{aligned}
& \left\|x_{k+1}-z\right\| \\
= & \left\|\left(I-\alpha_{k} \mu \mathcal{F}\right) z_{k}^{N}-\left(I-\alpha_{k} \mu \mathcal{F}\right) z-\alpha_{k} \mu \mathcal{F} z\right\| \\
\leq & \left\|\left(I-\alpha_{k} \mu \mathcal{F}\right) z_{k}^{N}-\left(I-\alpha_{k} \mu \mathcal{F}\right) z\right\|+\left\|\alpha_{k} \mu \mathcal{F} z\right\| \\
\leq & \left(1-\alpha_{k} \tau\right)\left\|z_{k}^{N}-z\right\|+\alpha_{k} \tau\left(\frac{\mu}{\tau}\|\mathcal{F} z\|\right) \\
\leq & \max \left\{\left\|z_{k}^{N}-z\right\|, \frac{\mu}{\tau}\|\mathcal{F} z\|\right\} \\
\leq & \max \left\{\left\|x_{k}-z\right\|, \frac{\mu}{\tau}\|\mathcal{F} z\|\right\} \\
\leq & \max \left\{\left\|x_{k-1}-z\right\|, \frac{\mu}{\tau}\|\mathcal{F} z\|\right\} \\
\vdots & \max \left\{\left\|x_{0}-z\right\|, \frac{\mu}{\tau}\left\|\mathcal{F}_{z}\right\|\right\}, k \geq 0,
\end{aligned}
$$

where $\tau=1-\sqrt{1-\mu\left(2 \eta-\mu L^{2}\right)} \in(0,1)$. Therefore, the sequence $\left\{x_{k}\right\}$ is bounded and so are the sequences 
$\left\{z_{k}^{i}\right\}$ and $\left\{\mathcal{F}_{k}^{i}\right\}, i=1,2, \ldots, N$. Without loss of generality, we can assume that they are bounded by a positive constant $M_{1}$. So the existence of $M_{1}$ is proved.

Step 2. We will prove that

$$
\left\|x_{k+1}-x^{*}\right\|^{2} \leq\left(1-b_{k}\right)\left\|x_{k}-x^{*}\right\|+b_{k} c_{k},
$$

where $\left\{b_{k}\right\}$ and $\left\{c_{k}\right\}$ are sequences of real numbers, and $x^{*}$ is the unique solution of (VIP).

By using Step 3 in Algorithm 1 and Lemma 1, we estimate the value of $\left\|x_{k+1}-x^{*}\right\|^{2}$ as follows

$$
\begin{aligned}
& \left\|x_{k+1}-x^{*}\right\|^{2} \\
& =\left\langle\left(I-\alpha_{k} \mu \mathcal{F}\right) z_{k}^{N}-x^{*}, x_{k+1}-x^{*}\right\rangle \\
& =\left\langle\left(I-\alpha_{k} \mu \mathcal{F}\right) z_{k}^{N}-\left(I-\alpha_{k} \mu \mathcal{F}\right) x^{*}, x_{k+1}-x^{*}\right\rangle \\
& \quad+\alpha_{k} \mu\left\langle\mathcal{F} x^{*}, x^{*}-x_{k+1}\right\rangle \\
& \leq\left(1-\alpha_{k} \tau\right)\left\|z_{k}^{N}-x^{*}\right\|\left\|x_{k+1}-x^{*}\right\| \\
& \quad+\alpha_{k} \mu\left\langle\mathcal{F} x^{*}, x^{*}-x_{k+1}\right\rangle \\
& \leq\left(1-\alpha_{k} \tau\right) \frac{\left\|z_{k}^{N}-x^{*}\right\|^{2}+\left\|x_{k+1}-x^{*}\right\|^{2}}{2} \\
& \quad+\alpha_{k} \mu\left\langle\mathcal{F} x^{*}, x^{*}-x_{k+1}\right\rangle .
\end{aligned}
$$

This implies that

$$
\begin{aligned}
\left\|x_{k+1}-x^{*}\right\|^{2} & \\
\leq & \frac{1-\alpha_{k} \tau}{1+\alpha_{k} \tau}\left\|z_{k}^{N}-x^{*}\right\|^{2}+\frac{2 \alpha_{k} \mu}{1+\alpha_{k} \tau}\left\langle\mathcal{F} x^{*}, x^{*}-x_{k+1}\right\rangle \\
= & \left(1-\frac{2 \alpha_{k} \tau}{1+\alpha_{k} \tau}\right)\left\|z_{k}^{N}-x^{*}\right\|^{2} \\
& \quad+\frac{2 \alpha_{k} \tau}{1+\alpha_{k} \tau}\left(\frac{\mu}{\tau}\left\langle\mathcal{F} x^{*}, x^{*}-x_{k+1}\right\rangle\right) .
\end{aligned}
$$

It follows from the condition (C3), Step 3 in Algorithm 1 and Lemma 3 that

$$
\begin{aligned}
& \left\|z_{k}^{N}-x^{*}\right\|^{2} \\
= & \left\|\left(1-\gamma_{k}^{N}\right)\left(z_{k}^{N-1}-x^{*}\right)+\gamma_{k}^{N}\left(\mathcal{J}_{\beta_{k}^{N}}^{\mathcal{A}_{N}^{N}} z_{k}^{N-1}-x^{*}\right)\right\|^{2} \\
\leq & \left(1-\gamma_{k}^{N}\right)\left\|z_{k}^{N-1}-x^{*}\right\|^{2}+\gamma_{k}^{N}\left\|\mathcal{J}_{\beta_{k}^{N}}^{\mathcal{A}_{N}} z_{k}^{N-1}-x^{*}\right\|^{2} \\
& \quad-\gamma_{k}^{N}\left(1-\gamma_{k}^{N}\right) \varphi\left(\left\|z_{k}^{N-1}-\mathcal{J}_{\beta_{k}^{N}}^{\mathcal{A}_{N}} z_{k}^{N-1}\right\|\right) \\
\leq & \left(1-\gamma_{k}^{N}\right)\left\|z_{k}^{N-1}-x^{*}\right\|^{2}+\gamma_{k}^{N}\left\|z_{k}^{N-1}-x^{*}\right\|^{2} \\
& \quad-\gamma_{k}^{N}\left(1-\gamma_{k}^{N}\right) \varphi\left(\left\|z_{k}^{N-1}-\mathcal{J}_{\beta_{k}^{N}}^{\mathcal{A}_{N}} z_{k}^{N-1}\right\|\right) \\
\leq & \left\|z_{k}^{N-1}-x^{*}\right\|^{2}-a(1-b) \varphi\left(\left\|z_{k}^{N-1}-\mathcal{J}_{\beta_{k}^{N}}^{\mathcal{A}_{N}} z_{k}^{N-1}\right\|\right)
\end{aligned}
$$

$$
\begin{aligned}
& \leq\left\|z_{k}^{0}-x^{*}\right\|^{2}-a(1-b) \sum_{i=1}^{N} \varphi\left(\left\|z_{k}^{i-1}-\mathcal{J}_{\beta_{k}^{i}}^{\mathcal{A}} z_{k}^{i-1}\right\|\right) \\
& =\left\|x_{k}-x^{*}\right\|^{2}-a(1-b) \sum_{i=1}^{N} \varphi\left(\left\|z_{k}^{i-1}-\mathcal{J}_{\beta_{k}^{i}}^{\mathcal{A}_{i}} z_{k}^{i-1}\right\|\right) .
\end{aligned}
$$

where $\varphi: \mathbb{R}^{+} \rightarrow \mathbb{R}^{+}$is a continuous strictly increasing and convex function with $\varphi(0)=0$. From (6) and (7) we obtain that

$$
\begin{aligned}
& \left\|x_{k+1}-x^{*}\right\|^{2} \\
& \leq\left(1-\frac{2 \alpha_{k} \tau}{1+\alpha_{k} \tau}\right)\left\|x_{k}-x^{*}\right\|^{2}+\frac{2 \alpha_{k} \tau}{1+\alpha_{k} \tau}\left(\frac{\mu}{\tau}\left\langle\mathcal{F} x^{*}, x^{*}-x_{k+1}\right\rangle\right)
\end{aligned}
$$

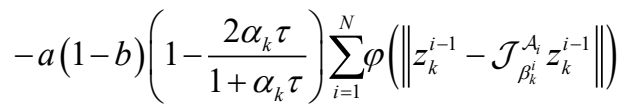

$$
\begin{aligned}
& \leq\left(1-\frac{2 \alpha_{k} \tau}{1+\alpha_{k} \tau}\right)\left\|x_{k}-x^{*}\right\|^{2}+\frac{2 \alpha_{k} \tau}{1+\alpha_{k} \tau}\left[\frac{\mu}{\tau}\left\langle\mathcal{F} x^{*}, x^{*}-x_{k+1}\right\rangle\right. \\
& \left.+a(1-b) \sum_{i=1}^{N} \varphi\left(\left\|z_{k}^{i-1}-\mathcal{J}_{\beta_{k}^{i}}^{\mathcal{A}_{i}} z_{k}^{i-1}\right\|\right)\right] .
\end{aligned}
$$

Putting

$$
\begin{aligned}
& b_{k}=\frac{2 \alpha_{k} \tau}{1+\alpha_{k} \tau} \\
& c_{k}=\frac{\mu}{\tau}\left\langle\mathcal{F} x^{*}, x^{*}-x_{k+1}\right\rangle+a(1-b) \sum_{i=1}^{N} \varphi\left(\left\|z_{k}^{i-1}-\mathcal{J}_{\beta_{k}^{\prime}}^{\mathcal{A}_{i}} z_{k}^{i-1}\right\|\right)
\end{aligned}
$$$$
\text { inequality (9) can be rewritten as (5). }
$$

Step 3. We claim that $\lim _{k \rightarrow \infty}\left\|x_{k}-x^{*}\right\|=0$, where $x^{*}$ is the unique solution of the (VIP).

We consider two cases.

Case 1. There exists an integer $k_{0} \geq 0$ such that $\left\|x_{k+1}-x^{*}\right\| \leq\left\|x_{k}-x^{*}\right\| \quad$ for all $k \geq k_{0}$. Then, $\lim _{k \rightarrow \infty}\left\|x_{k}-x^{*}\right\|$ exists. It then from (8) and (C1) that $0 \leq d_{k}$

$$
\begin{aligned}
& \leq\left(\left\|x_{k}-x^{*}\right\|^{2}-\left\|x_{k+1}-x^{*}\right\|^{2}\right) \\
& \quad+b_{k}\left(\left\langle\mathcal{A} x^{*}, x^{*}-x_{k+1}\right\rangle-\left\|x_{k}-x^{*}\right\|^{2}\right) \rightarrow 0
\end{aligned}
$$

as $k \rightarrow \infty$, where

$$
d_{k}=a(1-b)\left(1-b_{k}\right) \sum_{i=1}^{N} \varphi\left(\left\|z_{k}^{i-1}-\mathcal{J}_{\beta_{k}^{i}}^{\mathcal{A}_{i}} z_{k}^{i-1}\right\|\right),
$$

which implies that

$$
\left\|z_{k}^{i-1}-\mathcal{J}_{\beta_{k}^{i}}^{\mathcal{A}_{i}} z_{k}^{i-1}\right\| \rightarrow 0 \forall i=1,2, \ldots, N .
$$

Next, we will show that $\left\|x_{k}-\mathcal{J}_{\beta}^{\mathcal{A}_{i}} x_{k}\right\| \rightarrow 0$ for all $i=1,2, \ldots, N$. Indeed, in the case that $i=1$, from Steps 2 and 3 in Algorithm 1 and (10), we have

$$
\left\|x_{k}-\mathcal{J}_{\beta_{k}^{\mathcal{A}_{1}}}^{\mathcal{A}_{k}} x_{k}\right\|=\left\|z_{k}^{0}-\mathcal{J}_{\beta_{k}^{\prime}}^{\mathcal{A}_{1}} z_{k}^{0}\right\| \rightarrow 0 \text { as } k \rightarrow \infty .
$$


In the case that $i=2$, from the nonexpansive property of $\mathcal{J}_{\beta_{k}^{2}}^{\mathcal{A}_{2}}$, Steps 2 and 3 in Algorithm 1, and (10), we have

$$
\begin{aligned}
& \left\|x_{k}-\mathcal{J}_{\beta_{k}^{2}}^{\mathcal{A}_{2}} x_{k}\right\| \\
\leq & \left\|x_{k}-z_{k}^{1}\right\|+\left\|z_{k}^{1}-\mathcal{J}_{\beta_{k}^{2}}^{\mathcal{A}_{2}} z_{k}^{1}\right\|+\left\|\mathcal{J}_{\beta_{k}^{2}}^{\mathcal{A}_{2}} z_{k}^{1}-\mathcal{J}_{\beta_{k}^{2}}^{\mathcal{A}_{2}} x_{k}\right\| \\
\leq & 2\left\|x_{k}-z_{k}^{1}\right\|+\left\|z_{k}^{1}-\mathcal{J}_{\beta_{k}^{2}}^{\mathcal{A}_{2}} z_{k}^{1}\right\| \\
\leq & 2\left\|z_{k}^{0}-\mathcal{J}_{\beta_{k}^{1}}^{\mathcal{A}_{1}} z_{k}^{0}\right\|+\left\|z_{k}^{1}-\mathcal{J}_{\beta_{k}^{2}}^{\mathcal{A}_{2}} z_{k}^{1}\right\| \rightarrow 0 \text { as } k \rightarrow \infty .
\end{aligned}
$$

Similarly, we obtain $\left\|x_{k}-\mathcal{J}_{\beta_{k}^{\prime}}^{\mathcal{A}_{i}} x_{k}\right\| \rightarrow 0$ for all $i=3,4, \ldots, N$. It follows from the condition (C3) and Lemma 5 that

$$
\left\|x_{k}-\mathcal{J}_{\beta}^{\mathcal{A}_{i}} x_{k}\right\| \leq 2\left\|x_{k}-\mathcal{J}_{\beta_{k}^{\prime}}^{\mathcal{A}_{i}} x_{k}\right\|
$$

for all $i=1,2, \ldots, N$. So

$$
\left\|x_{k}-\mathcal{J}_{\beta}^{\mathcal{A}_{i}} x_{k}\right\| \rightarrow 0 \forall i=1,2, \ldots, N .
$$

Now, we will show that $\limsup c_{k} \leq 0$. Indeed, suppose that $\left\{x_{k_{n}}\right\}$ is a subsequence of $\left\{x_{k}\right\}$ such that

$$
\limsup _{k \rightarrow \infty}\left\langle\mathcal{F} x^{*}, x^{*}-x_{k}\right\rangle=\lim _{n \rightarrow \infty}\left\langle\mathcal{F} x^{*}, x^{*}-x_{k_{n}}\right\rangle .
$$

Since $\left\{x_{k_{n}}\right\} \quad$ is bounded, there exists a subsequence $\left\{x_{k_{n_{m}}}\right\}$ of $\left\{x_{k_{n}}\right\}$ which converges weakly to some point $x^{+}$. Without loss of generality, we may assume that $x_{k_{n}} \rightarrow x^{+}$. We shall prove that $x^{+} \in \mathcal{C}$. Indeed, from Lemma 2 and (11) we obtain $x^{+} \in \operatorname{Fix}\left(\mathcal{J}_{\beta}^{\mathcal{A}_{i}}\right)$ for all $i=1,2, \ldots, N$. It follows from Lemma 4 that $x^{+} \in \mathcal{C}$. Since $x^{*}$ is the unique solution of (VIP), $\left\langle\mathcal{F}^{*}, x^{*}-x^{+}\right\rangle \leq 0$, which combines with (12), we get

$$
\limsup _{k \rightarrow \infty}\left\langle\mathcal{F} x^{*}, x^{*}-x_{k}\right\rangle \leq 0 .
$$

Putting $M_{2}=\sup _{k}\left\|\mathcal{F} x_{k}\right\|$, from Steps 2 and 3 in Algorithm 1, the condition (C4), and Lemma 1 we have

$$
\begin{aligned}
& \left\|x_{k+1}-x_{k}\right\| \\
& =\left\|\left(I-\alpha_{k} \mu \mathcal{F}\right) z_{k}^{N}-x_{k}\right\| \\
& \leq\left\|\left(I-\alpha_{k} \mu \mathcal{F}\right) z_{k}^{N}-\left(I-\alpha_{k} \mu \mathcal{F}\right) x_{k}\right\|+\alpha_{k} \mu M_{2} \\
& \leq\left\|x_{k}-\left(1-\gamma_{k}^{N}\right) z_{k}^{N-1}-\gamma_{k}^{N} \mathcal{J}_{\beta_{k}^{N}}^{\mathcal{A}_{i}} z_{k}^{N-1}\right\|+\alpha_{k} \mu M_{2} \\
& \leq\left\|x_{k}-z_{k}^{N-1}\right\|+\left\|z_{k}^{N-1}-\mathcal{J}_{\beta_{k}^{N}}^{\mathcal{A}_{k}} z_{k}^{N-1}\right\|+\alpha_{k} \mu M_{2} \\
& \leq\left\|x_{k}-\left(1-\gamma_{k}^{N-1}\right) z_{k}^{N-2}-\gamma_{k}^{N-1} \mathcal{J}_{\beta_{k}^{N-1}}^{\mathcal{A}_{\mathcal{A}}} z_{k}^{N-2}\right\|
\end{aligned}
$$

$$
+\left\|z_{k}^{N-1}-\mathcal{J}_{\beta_{k}^{N}}^{\mathcal{A}_{N}} z_{k}^{N-1}\right\|+\alpha_{k} \mu M_{2}
$$

$\leq \sum_{i=1}^{N}\left\|z_{k}^{i-1}-\mathcal{J}_{\beta_{k}^{i}}^{\mathcal{A}_{i}} z_{k}^{i-1}\right\|+\alpha_{k} \mu M_{2}$.

It follows from (C1), (10), and (14) that $\left\|x_{k+1}-x_{k}\right\| \rightarrow 0$ as $k \rightarrow \infty$. Thus, by (13) we have

$$
\limsup _{k \rightarrow \infty}\left\langle\mathcal{F} x^{*}, x^{*}-x_{k+1}\right\rangle \leq 0 \text {, and } \limsup _{k \rightarrow \infty} c_{k} \leq 0 .
$$

Hence, all conditions of Lemma 7 for (5) are satisfied. Therefore, we immediately deduce that $x_{k} \rightarrow x^{*}$ as $k \rightarrow \infty$.

Case 2. There exists a subsequence $\left\{k_{i}\right\}$ of $\{k\}$ such that $\left\|x_{k_{i}}-x^{*}\right\| \leq\left\|x_{k_{i}+1}-x^{*}\right\|$ for all $i \geq 0$. Hence, by Lemma 6 , there exists an integer, nondecreasing sequence $\{v(k)\}$ for all $k \geq k_{0}$ (for some $k_{0}$ large enough) such that $v(k) \rightarrow \infty$ as $k \rightarrow \infty$,

$$
\begin{aligned}
& \left\|x_{v(k)}-x^{*}\right\| \leq\left\|x_{v(k)+1}-x^{*}\right\|, \\
& \left\|x_{k}-x^{*}\right\| \leq\left\|x_{v(k)+1}-x^{*}\right\|
\end{aligned}
$$

for each $k \geq 0$. From (8), the condition (C1), and the first inequality in (15), we have

$$
0 \leq d_{v(k)} \leq b_{v(k)}\left(\left\langle\mathcal{F} x^{*}, x^{*}-x_{v(k)+1}\right\rangle-\left\|x_{v(k)}-x^{*}\right\|^{2}\right) \rightarrow 0
$$

By a similar argument to Case 1, we obtain

$$
\left\|x_{v(k)}-\mathcal{J}_{\beta}^{\mathcal{A}_{i}} x_{v(k)}\right\| \rightarrow 0 \text { as } k \rightarrow \infty
$$

for all $i=1,2, \ldots, N$, and

$$
\limsup _{k \rightarrow \infty}\left\langle\mathcal{F}^{*}, x^{*}-x_{v(k)+1}\right\rangle \leq 0 .
$$

From (8), $\left\{b_{v(k)}\right\} \subset(0,1)$, and the first inequality in (15), we have also that

$$
\left\|x_{v(k)}-x^{*}\right\|^{2} \leq\left\langle\mathcal{F} x^{*}, x^{*}-x_{v(k)+1}\right\rangle .
$$

Thus,

$$
\lim _{k \rightarrow \infty}\left\|x_{v(k)}-x^{*}\right\|^{2}=0 .
$$

Finally, from (8) with $k$ replaced by $v(k)$, we can write that

$$
\begin{gathered}
\left\|x_{v(k)+1}-x^{*}\right\|^{2} \\
\leq\left(1-\frac{2 \alpha_{k} \tau}{1+\alpha_{k} \tau}\right)\left\|x_{v(k)}-x^{*}\right\|^{2}
\end{gathered}
$$




$$
\begin{aligned}
& +\frac{2 \alpha_{k} \tau}{1+\alpha_{k} \tau}\left(\frac{\mu}{\tau}\left\langle\mathcal{F} x^{*}, x^{*}-x_{v(k)+1}\right\rangle\right) \\
& -a(1-b)\left(1-\frac{2 \alpha_{k} \tau}{1+\alpha_{k} \tau}\right) \sum_{i=1}^{N} \varphi\left(\left\|z_{k}^{i-1}-\mathcal{J}_{\beta_{k}^{i}}^{\mathcal{A}_{i}} z_{k}^{i-1}\right\|\right)
\end{aligned}
$$

By virtue of (16), (17), and (C1), $\lim _{k \rightarrow \infty}\left\|x_{v(k)+1}-x^{*}\right\|^{2}=0$, which together with the second inequality in (15) implies that $\lim _{k \rightarrow \infty}\left\|x_{k}-x^{*}\right\|=0$. The proof is completed.

\section{Numerical Results}

To illustrate Theorem 1, we consider the following examples. We perform the iterative schemes in MATLAB 2021a running on a laptop with Intel(R) Core(TM) i5-1135G7 CPU @ 2.40GHz 2.42GHz, 8GB RAM. Some signs in the result table:

- $k$ : Number of iterative steps.

- $x_{0}$ : The initial guess.

- $x_{k}$ : Solution in $k$-th step.

Now, with the purpose of illustrating the convergence of the Algorithm 1, we will apply the algorithm to solve a optimization problem over the set of common zero points of $N$ maximal monotone operators. Consider the following optimization problem: find a point $x^{*} \in \mathcal{C}$ such that

$$
\varphi\left(x^{*}\right)=\min _{x \in \mathcal{C}} \varphi(x), \quad \mathcal{C}=\bigcap_{I=1}^{N} C_{i} \neq \varnothing
$$

where $\varphi(x)$ is a convex function having a strongly monotone and Lipschitz continuous derivative $\nabla \varphi(x)$ on Hilbert space $\mathcal{H}$, and $\mathcal{C}$ is a closed and convex subset of $\mathcal{H}$.

Example 1. Let $\mathcal{H}=\mathbb{R}^{5}$. Here, we choose $\mathcal{A}_{i}: \mathbb{R}^{5} \rightarrow \mathbb{R}^{5}, i=1,2, \ldots, N$, defined by

$$
\begin{aligned}
& \mathcal{A}_{i}(x)=\left(\begin{array}{ccccc}
0 & 0 & 0 & 0 & 0 \\
0 & i & i & 0 & 0 \\
0 & -i & i & 0 & 0 \\
0 & 0 & 0 & i & -i \\
0 & 0 & 0 & 0 & i
\end{array}\right)\left(\begin{array}{c}
x_{1} \\
x_{2} \\
x_{3} \\
x_{4} \\
x_{5}
\end{array}\right) \\
& x=\left(x_{1}, x_{2}, x_{3}, x_{4}, x_{5}\right)^{\mathrm{T}} \in \mathbb{R}^{5}
\end{aligned}
$$

that is a maximal monotone operator, the function $\varphi$ defined by $\varphi(u)=u^{T} \mathcal{A} u+b^{T} u+c$, where

$$
\mathcal{A}=\left(\begin{array}{lllll}
3 & 0 & 0 & 0 & 0 \\
0 & 3 & 0 & 0 & 0 \\
0 & 0 & 3 & 0 & 0 \\
0 & 0 & 0 & 3 & 0 \\
0 & 0 & 0 & 0 & 3
\end{array}\right), b=\left(\begin{array}{c}
-6 \\
2 \\
0 \\
1 \\
5
\end{array}\right) \text { and } c=5
$$

with

$$
\begin{gathered}
\mathcal{F} x=\nabla \varphi(x)=\left(\begin{array}{c}
6 x_{1}-6 \\
6 x_{2}+2 \\
6 x_{3} \\
6 x_{4}+1 \\
6 x_{5}+5
\end{array}\right) \\
x=\left(x_{1}, x_{2}, x_{3}, x_{4}, x_{5}\right)^{\mathrm{T}} \in \mathbb{R}^{5}
\end{gathered}
$$

are 6-strongly monotone and 6-Lipschitz continuous on Euclidean space $\mathbb{R}^{5}$.

The common zeros set of $\mathcal{A}_{i}, i=1,2, \ldots, N$, is

$$
\begin{aligned}
\mathcal{C}: & =\bigcap_{i=1}^{N} \operatorname{Zer}\left(\mathcal{A}_{i}\right) \\
= & \left\{u=\left(u_{1}, u_{2}, u_{3}, u_{4}, u_{5}\right)^{T} \in \mathbb{R}^{5} \mid\right. \\
& \left.u_{2}=u_{3}=u_{4}=u_{5}=0\right\} .
\end{aligned}
$$

The unique solution of variational inequality problem

$$
\left\langle\nabla \varphi\left(x^{*}\right), x-x^{*}\right\rangle \geq 0 \text { for all } x \in \mathcal{C}
$$

is $x^{*}=(1,0,0,0,0)^{\mathrm{T}} \in \mathbb{R}^{5}$. It is well known that (see [11], Propositions 5.1 and 5.2) the variational inequality problem (19) is equivalent to the optimization problem (18).

We choose, for each $k$

$$
\alpha_{k}=\frac{1}{k}, \beta_{k}^{i}=\frac{5 i+1}{i+1}, \gamma_{k}^{i}=(i+1)^{-1 / 5},
$$

where $i=1,2, \ldots, 20$, and in the first tests, we consider the case when $\mu=1 / 6$. The numerical results for the Algorithm 1 with initial point $x_{0}=(0,5,1,-1,0)^{\mathrm{T}} \in \mathbb{R}^{5}$ is shown in Table 1.

In another run of tests for the Algorithm 1, we consider the case when $\mu=1 / 6, \quad \beta_{k}^{i}=\sqrt{2 i+1}$, $\alpha_{k}=\sum_{n=1}^{k} \frac{(-1)^{n+1}}{200 n}$ and $\gamma_{k}^{i}=\sum_{n=1}^{k} \frac{(-1)^{n+1}}{2 n-1}$ for each $k$ and $i=1,2, \ldots, 10$. These sequences are contained in $(0,1)$ but $\sum_{k=1}^{\infty}\left|\alpha_{k+1}-\alpha_{k}\right|=\infty$ and $\sum_{k=1}^{\infty}\left|\gamma_{k+1}^{i}-\gamma_{k}^{i}\right|=\infty$. The next two tables display the numerical results obtained for the initial guesses $x_{0}=(-3,1,2,5,-4)^{\mathrm{T}} \quad$ and $x_{0}=(100,20,50,-70,-90)^{\mathrm{T}} \in \mathbb{R}^{5} \quad, \quad$ respectively (Tables 2 and 3). 
JST: Smart Systems and Devices

Volume 31, Issue 2, September 2021, 117-124

Table 1. Numerical results for Algorithm 1 with the initial guess $x_{0}=(0,5,1,-1,0)^{T} \in \mathbb{R}^{5}$.

\begin{tabular}{rccccc}
\hline $\operatorname{Iter}(k)$ & 100 & 500 & 1000 & 1500 & 2000 \\
\hline$x_{k}^{1}$ & 1.0000 & 1.0000 & 1.0000 & 1.0000 & 1.0000 \\
$x_{k}^{2}$ & -0.0033 & -0.0007 & -0.0003 & -0.0002 & -0.0002 \\
$x_{k}^{3}$ & -0.0000 & -0.0000 & -0.0000 & -0.0000 & -0.0000 \\
$x_{k}^{4}$ & -0.0017 & -0.0003 & -0.0002 & -0.0001 & -0.0001 \\
$x_{k}^{5}$ & -0.0083 & -0.0017 & -0.0008 & -0.0006 & -0.0004 \\
\hline$\left\|x_{k}-x^{*}\right\|$ & 0.0091 & 0.0018 & $9.1284 \times 10^{-4}$ & $6.0858 \times 10^{-4}$ & $4.4644 \times 10^{-4}$ \\
\hline
\end{tabular}

Table 2. Numerical results for Algorithm 1 with the initial guess $x_{0}=(-3,1,2,5,-4)^{\top} \in \mathbb{R}^{5}$.

\begin{tabular}{rccccc}
\hline $\operatorname{Iter}(k)$ & 100 & 500 & 1000 & 1500 & 2000 \\
\hline$x_{k}^{1}$ & -1.8240 & 0.8759 & 0.9961 & 0.9999 & 1.0000 \\
$x_{k}^{2}$ & -0.0011 & -0.0012 & -0.0012 & -0.0012 & -0.0012 \\
$x_{k}^{3}$ & -0.0000 & -0.0000 & -0.0000 & -0.0000 & -0.0000 \\
$x_{k}^{4}$ & -0.0006 & -0.0006 & -0.0006 & -0.0006 & -0.0006 \\
$x_{k}^{5}$ & -0.0029 & -0.0029 & -0.0029 & -0.0029 & -0.0029 \\
\hline$\left\|x_{k}-x^{*}\right\|$ & 2.8240 & 0.1241 & 0.0050 & 0.0032 & 0.0032
\end{tabular}

Table 3. Numerical results for Algorithm 1 with the initial guess $x_{0}=(100,20,50,-70,-90)^{\top} \in \mathbb{R}^{5}$.

\begin{tabular}{rccccc}
\hline $\operatorname{Iter}(k)$ & 100 & 500 & 1000 & 1500 & 2000 \\
\hline$x_{k}^{1}$ & 70.8944 & 4.0722 & 1.0954 & 1.0030 & 1.0001 \\
$x_{k}^{2}$ & -0.0011 & -0.0012 & -0.0012 & -0.0012 & -0.0012 \\
$x_{k}^{3}$ & -0.0000 & -0.0000 & -0.0000 & -0.0000 & -0.0000 \\
$x_{k}^{4}$ & -0.0006 & -0.0006 & -0.0006 & -0.0006 & -0.0006 \\
$x_{k}^{5}$ & -0.0029 & -0.0029 & -0.0029 & -0.0029 & -0.0029 \\
\hline$\left\|x_{k}-x^{*}\right\|$ & 69.8944 & 3.0722 & 0.0955 & 0.0043 & 0.0032 \\
\hline
\end{tabular}

\section{Remark 1.}

- The initial guess $x_{0}, \alpha_{k}, \beta_{k}$ and $\gamma_{k}$ affect on the number of iterations $k$.

- The sequence $\left\{\alpha_{k}\right\}$ doesn't satisfy the condition (C2), the sequence $\left\{x_{k}\right\}$ generated by Algorithm 1 converges strongly to the unique solution of the problem but the sequence $\left\{x_{n}\right\}$ generated by Theorem 1 (see [12]) doesn't converge strongly.

\section{Conclusion}

The paper has given the following issues:

We prove the strong convergence of the iterative method.

- We give an application for the optimization problem and calculate a numerical example that 
JST: Smart Systems and Devices

Volume 31, Issue 2, September 2021, 117-124

illustrate the convergence of method in a Hilbert space.

\section{Reference}

[1]. Rockafellar R.T., Monotone operators and the proximal point algorithm, SIAM J. Control Optim., vol. 14 , no. 5 , pp. $877-898,1976$. https://doi.org/10.1137/0314056

[2]. Takahashi W., Convex Analysis and Approximation of Fixed Points, Yokohama Publishers, Yokohama, 2000 (4-946552-04-9: Japanese).

[3]. Yamada Y., The hybrid steepest-descent method for variational inequalities problems over the intersection of the fixed point sets of nonexpansive mappings, Edited by Butnariu D., Censor Y., Reich S., Inhently Parallel Algorithms in Feasibility and Optimization and Their Applications, North-Holland, Amsterdam, Holland, pp. 473-504, 2001. https://doi.org/10.1016/S1570-579X(01)80028-8

[4]. Ceng L.C., Ansari Q.H., Yao J.C., Mann-type steepsetdescent and modified hybrid steepset-descent methods for variational inequality in Banach spaces, Numer. Funct. Anal. Optim., vol. 29, pp. 987-1033, 2008. https://doi.org/10.1080/01630560802418391

[5]. Opial Z., Weak convergence of the sequence of successive approximations for nonexpansive mappings, Bull. Am. Math. Soc., vol. 73, no. 4, pp. 591-597, 1967. https://doi.org/10.1090/S0002-9904-1967-11761-0

[6]. $\mathrm{Xu}$ H.K., Inequalities in Banach spaces with applications, Nonlinear Anal., vol. 16, no. 12, pp. 1127 1138, 1991.
https://doi.org/10.1016/0362-546X(91)90200-K

[7]. Reich S., Extension problems for accretive sets in Banach spaces, J. Funct. Anal., vol. 26, pp. 378-395, 1977.

https://doi.org/10.1016/0022-1236(77)90022-2

[8]. Tuyen T.M., Thuy Ng.T.T., Trang Ng.M., A strong convergence theorem for a parallel iterative method for solving the split common null point problem in Hilbert spaces, J. Optim. Theory Appl., vol. 183, no. 2, pp. 271291, 2019.

https://doi.org/10.1007/s10957-019-01523-w

[9]. Maingé P.E., Strong convergence of projected subgradient methods for nonsmooth and nonstrictly convex minimization, Set-Valued Anal., vol. 16, no. 78, pp. 899-912, 2008.

https://doi.org/10.1007/s11228-008-0102-z

[10].Xu H.K., Strong convergence of an iterative method for nonexpansive and accretive operators, J. Math. Anal. Appl., vol. 314, pp. 631- 643, 2006. https://doi.org/10.1016/j.jmaa.2005.04.082

[11].Kinderlerhrer D., Stampacchia G., An Introduction to Variational Inequalities and Their Applications, Academic Press, New York, 1980, pp. 22-104. https://epubs.siam.org/doi/book/10.1137/1.978089871 9451?mobileUi $=0$

[12].Kim J.K., Tuyen T.M., New Iterative methods for finding a common zero of a finite family of monotone operators in Hilbert spaces, Bull. Korean Math. Soc., vol 54, no 4, pp 1347-1359, 2017. http://www.koreascience.or.kr/article/JAKO20172383 9750277.page 